\title{
Enantioselective Extraction of Borate Complexed- Diol Racemates
}

\author{
R. Mathur \\ Department of Chemistry, Jai Naraian Vyas University, Jodhpur-342008 (India)
}

\begin{abstract}
The use of liquid-liquid extraction for enantioseparation has been known since 1959, although the first articles in the English literature appeared in the late 1960's. Enantioselective liquid-liquid extraction (ELLE) is an attractive alternative for chromatography and resolution by crystallization, although judged from the scientific literature, commercialization of ELLE has not been achieved to date. This study reports ELLE of racemates with diol or those capable of having diol type of structure. Such compounds are borate complexed followed by extraction into non polar phase using a chiral quaternary ammonium salt. Ion pair formed by the chiral quaternary ammonium salt with borate complexed diol type racemate exhibit differential transportation behavior. Selectively extracted ion-pair is labile under acidic pH. Hence, one of the two enantiomer is recovered in larger excess as compared with another one from organic phase.
\end{abstract}

Keywords: Enantiomers, enantioseparation, Borate Complexed- Diol Racemates, Chiral phase transfer catalysts ,Benzylquininium Chloride

\section{Introduction}

The need of an enantiopure compounds is of great importance for the pharmaceutical ${ }^{1}$, agrochemical, flavour and fragrances industries. ${ }^{2}$ They may be obtained either via a synthetic approach, ${ }^{3}$ or via the separation of racemates. ${ }^{4,5}$ The synthetic routes can be based on natural compounds, fermentation or asymmetric synthesis. Enantiopure compounds may also be obtained by racemic synthesis followed by enantiomer separation. A facile synthesis of racemic compounds followed by a racemate separation using a broadly applicable technology that is easily developed is possibly very beneficial. Separation of racemates on industrial scale is usually done using resolution by crystallization. $^{6-10}$. Besides, chromatographic techniques have also been developed and employed for chiral separations. However, for scaling up of successful and very broadly applicable chromatography high capital investments are required.

Phase transfer catalysis was developed to facilitate the reaction between reactants situated in two different, immiscible phases ${ }^{11-12}$. Quaternary ammonium compounds have extensively been used for this purpose. Chiral phase transfer catalysts (Chiral PTC) were synthesized and used for stereoselective synthesis. ${ }^{13-15}$ It has been observed that when a two phase reaction is carried out in presence of chiral PTC, one isomer of product is formed in excess. However, studies have revealed that formation of one enantiomer in excess is not a real sense stereo selective synthesis, but it is a kinetic resolution. ${ }^{16}$

It is therefore, considered that instead of using chiral PTC for the purpose of synthesis, these should be used for resolution through selective extraction in normal achiral solvents. The chiral quaternary ammonium salt forms dissociable diastereoisomers with racemates. Due to difference in physical properties; diastereoisomer of one of the two enantiomer is selectively extracted from one phase into another at a faster rate than that of the other enantiomer.
Mandelicacid, a $\alpha$-hydroxy acid which behaves like 1,2dihydroxy compounds is chosen for the present study. It forms a cyclic complex with boric acid under alkaline condition. The resulting complex has a tetrahedral structure and is anionic. ${ }^{17-18}$ Chiral quaternary ammonium ion form salt with borate complexed mandelic acid.

\section{Experimental}

Preparation of Benzylquininium Chloride:BenzylquininiumChloride is prepared by simple quaternization reaction. $7.8 \mathrm{~g}(0.01 \mathrm{~mole})$ of quinine sulphatewas converted to soluble quinine chloride by treatment with $2.4 \mathrm{~g}(0.01 \mathrm{~mole})$ of barium chloride dissolved in $150 \mathrm{~mL}$ of water. Quinine hydrochloride was separated from insoluble barium sulphate by simple filtration. Free quinine base was recovered from solution by neutralizing with $0.8 \mathrm{~g}(0.02$ mole $)$ of sodium hydroxide. This resulted in precipitation of free quinine base which was filtered and dried. This was taken in a mixture of $80 \mathrm{~mL}$ benzene and 20 $\mathrm{mL}$ ethanol. $2.5 \mathrm{~g}(0.02$ mole $)$ of distilled benzyl chloride was added to the solution. Reaction mixture was refluxed for $24 \mathrm{~h}$. The solvent from the mixture was removed in a rotavapour and residue was washed with n-hexane to remove unreacted reagents. The resulting product was a greenish, highly fluorescent product which was characterized by IR spectral studies.

Stereo selective Extraction and determination of partition coefficient of mandelic acid- borate complex:An aqueous solution $1.5 \mathrm{~g}(0.01$ mole) of mandelic acid in $40 \mathrm{~mL}$ water containing $0.6 \mathrm{~g}$ ( 0.01 mole) of boric acid was made upto 50 $\mathrm{mL}$ by adding sodium hydroxide solution so that $\mathrm{pH}$ is maintained at $9.10 \mathrm{~mL}$ of this solution was drawn out and diluted to $200 \mathrm{~mL}$. To the remaining $40 \mathrm{~mL}$ of the solution 0.01 mole of benzylquininium Chloride, dissolved in $40 \mathrm{~mL}$ toluene was added. The solution was then stirred for $2-3 \mathrm{~h}$. After equilibriation, aqueous and organic phase were separated. $5 \mathrm{~mL}$ of aqueous phase was drawn out and diluted to $100 \mathrm{~mL}$. Concentration of mandelic acid in two solutions was determined by adding in excess of $0.01 \mathrm{M}$ of periodate 


\section{International Journal of Science and Research (IJSR) \\ ISSN (Online): 2319-7064}

Index Copernicus Value (2013): 6.14 | Impact Factor (2015): 6.391

with saturated sodium bicarbonate and freshly prepared 20\% potassium iodide solution. The liberated iodine was titrated with $0.01 \mathrm{~N}$ sodium arsenite solution. Partition coefficient calculated from these results was found to be 1.66.Enantioseparation was monitored by determining absorbance values at $\lambda$ max of $220 \mathrm{~nm}$ for mandelic acidafter acidification to dissociate borate complexed mandelic acid. For borate complexed mandelic acid $\lambda \max$ is shifted to a higher wavelength side i.e., $240 \mathrm{~nm}$.

\section{Result and Discussion}

Chiral quaternary ammonium salts have been used for a long for stereo selective synthesis of enantiomers. Mandelic acid is one of the most common racemate with behavior like that of the 1,2-dihydroxy compoundsforming borate complex under alkaline conditions. The stoichiometry of the boratediol complexes can either be 1:1 with two free $-\mathrm{OH}$ groups or a 1:2 spiro type. ${ }^{19-21}$ When forming an ion-pair with chiral benzylquininium chloride, one of the two borate complexed mandelic acid corresponding to two enantiomer make a relatively strong ion pair as compared to another one. This causes stereo selective difference in mobility and extraction of one of the two borate complexed enantiomerbenzylquininiumchloride ion pair into organic phase.

Davankov and et.al, ${ }^{22}$ have established that the formation constants (hence the thermodynamic stability) of borate complexes is large enough to give a good separation of diolborate complexes. Stereo selective formation of mandelic acid- borate complex and subsequent resolution on a chiral polygalactomannan has also been reported. Borate complexing for optical resolution of amino acids with diol type structure on polygalactomannan impregnated silica gel thin-layer chromatographic plates has also been reported by our group. ${ }^{23}$ Partition coefficient data and optical purity data of the experiment too suggest a promising stereo selective extraction through borate complexing.

Selective extraction was monitored by optical purity data which showed a maximum value of $82 \%$ for $(+)$ mandelic acid after equilibriation.

\section{References}

[1] Ager, D. J. Handbook of Chiral Chemicals; 2nd ed.; Marcel Dekker: New York, 2005.

[2] Ramos Tombo, G. M.; Bellus, D. Angew. Chem. Int. Ed. 1991, 30 (10), 1193-1215.

[3] Noyori, R. Asymmetric Catalysis in Organic Synthesis; Wiley: New York, 1994.

[4] Sheldon, R. A. Chirotechnology; Marcel Dekker: New York, 1993.

[5] Collins, A. N.; Sheldrake, G. N.; Crosby, J. Chirality in Industry II; New York:Wiley and Sons: 1997.

[6] Bruggink, A. Rational Design in Resolutions. In Chirality in Industry II, Collins, A. N., Ed.; Wiley: Chichester, 1997; 81-98.

[7] Faigl, F.; Fogassy, E.; Nogradi, M.; Palovics, E.; Schindler, J. Tetrahedron Asym. 2008, 19 (5), 519536.
[8] Kozma, D. CRC Handbook of Optical Resolutions via Diastereomeric Salt Formation; CRC Press LLC: Boca Raton: 2002.

[9] Fogassy, E.; Nogradi, M.; Kozma, D.; Egri, G.; Palovics, E.; Kiss, V. Org. Biomol. Chem. 2006, 4 (16), 3011-3030.

[10] Leeman, M.; Brasile, G.; Gelens, E.; Vries, T.; Kaptein, B.; Kellogg, R. Angew. Chem. Int. Ed. 2008, 47 (7), 1287-1290.

[11] Starks C.M. and Liotta C., "Phase Transfer Catalysis: Principles and Techniques", Academic Press, New York, 1978.

[12] Dehmlow E.V. and Dehmlow S.S., "Phase Transfer Catalysis", VerlagChemie, Weinheim, 1983.

[13] Saigo K., Koda H. and Nohiro H., Bull.Chem.Soc.Jpn., 1979, 52,3119

[14] Julia S., Ginebreda A., Guixer J., Masana J., Tomas A. and Colonna S., J. Chem. Soc. Perkin Trans., 1981,I,574

[15] Balcells J., Colonna S. and Fornasier R., Synthesis, 1976,266

[16] Julia S., Ginebreda A. and Guixer J., J. Chem. Soc. Chem. Commun., 1978, 742

[17] Mathur. R., Bohra S., Mathur V., Narang C.K., and Mathur N.K., Chromatographia, 1992,33(7/8), 336

[18] Mathur R., Ph.D. Thesis, " Studies on Separation Methods Based on Polysaccharide Gel and Phase Transfer Methods", University of Jodhpur, 1991

[19] Nickerson R.F., J. Inorg. Nucl. Chem., 1970,32,1400

[20] Yoshino K., Kotaka M., Okamoto M. and Kakihana H., Bull Chem. Soc. Jpn., 1979,52,3005

[21] Vanduin M., Peters J.A., Kieboom A.P.G. and Bekkum Van, Tetrahedron, 1985,1411

[22] Davankov V.A. and Zolotarev V.A., J.Chromatogr. $1978,155,285$

[23] Mathur. V., Kanoongo N., Mathur R., Narang C.K., and Mathur N.K., J. of Chromatography A, 19942,685, 360 